\title{
Ein blauer Brief für Antibiotika-Fans
}

\section{Zumindest in Großbitannien genügt ein einfacher Informationsbrief, um die Antibiotika-Verordnun- gen von „Hochverschreibern" zu verringern.}

_ Antibiotikaresistenzen sind auf dem Vormarsch - und leider spielen die bisweilen unnötigen Verordnungen in Hausarztpraxen bei dieser ungünstigen Entwicklung eine wichtige Rolle. Aus der Datenbank des britischen National Health Service (NHS) zog man für einen Versuch 1.581 allgemeinärztliche Praxen, die bei den Antibiotikaverordnungen im oberen Fünftel lagen. Am 29. September 2014 erhielt die Hälfte dieser Praxen, insgesamt 3.227 Allgemeinärzte, einen Brief, in dem auf diesen Umstand hingewiesen und die Bedeutung nicht indizierter Antibiotikaverordnungen für die Resistenzentwicklung geschildert wurde. Die andere Hälfte diente als Kontrollgruppe.

Zwischen Oktober 2014 und März 2015 betrug die Zahl der Antibiotikaverschreibungen der Interventionspraxen 126,98 je 1.000 Patienten, die der Kontrollpraxen 131,25. Dieser Unterschied von 4,27 Verordnungen (3,3\%) war statistisch hochsignifikant. Absolut gesehen wurden in der Interventionsgruppe 73.406 Antibiotikaverordnungen weniger ausgestellt.

Im Dezember 2014 wurde die Stichprobe in zwei Gruppen rerandomisiert. In einer wurden Plakate für das Wartezimmer und Info-Broschüren für Patienten verteilt. Mit dieser Maßnahme änderte sich die Antibiotikaverordnung zwischen Dezember 2014 und März 2015 nicht signifikant.

- Hallsworth M et al. Provision of social norm feedback to high prescribers of antibiotics in general practice: a pragmatic national randomised controlled trial. Lancet. 2016;387:1743-52

\section{KOMMENTAR}

Bei der Lektüre dieser Daten wird bei vielen deutschen Praxisärzten als erstes der Gedanke hochkochen, dass Ärzte hier als Versuchskaninchen missbraucht werden. So etwas ist wohl nur im staatlich gelenkten nationalen Gesundheitsdienst des Vereinigten Königreichs möglich. Vielleicht wird aber der eine oder andere etwas nachdenklich, wenn er erfährt, dass sich sein Verordnungsverhalten womöglich ganz erheblich von dem der Kollegen unterscheidet - und offensichtlich nicht der Untergang des Abendlandes eintritt, wenn man weniger Antibiotika verordnet.

Bei diesem Thema geht es einmal nicht um die oft ins Feld geführten Einsparungen der Krankenkassen, sondern um ein vitales gesundheitspolitisches Problem. Für den Verhaltensforscher ist interessant zu sehen, dass bereits eine minimale, extrem kostengünstige Intervention wie ein Brief zumindest kurzfristig das Verschreibungsverhalten eines Arztes signifikant beeinflussen kann.

Prof. Dr. med. H. S. FüeßI

\section{Hier steht eine Anzeige.}

\author{
Springer
}

